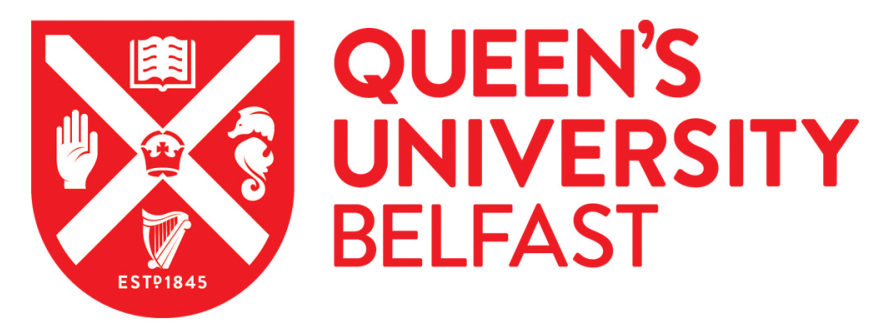

\title{
Pilot Power Control for Cell-free Massive MIMO
}

Mai, T. C., Ngo, H. Q., Egan, M., \& Duong, T. Q. (2018). Pilot Power Control for Cell-free Massive MIMO. IEEE Transactions on Vehicular Technology, 67(11), 11264 - 11268. https://doi.org/10.1109/TVT.2018.2867606

\author{
Published in: \\ IEEE Transactions on Vehicular Technology
}

\section{Document Version:}

Peer reviewed version

\section{Queen's University Belfast - Research Portal:}

Link to publication record in Queen's University Belfast Research Portal

\section{Publisher rights}

(c) 2018 IEEE.

This work is made available online in accordance with the publisher's policies. Please refer to any applicable terms of use of the publisher.

\section{General rights}

Copyright for the publications made accessible via the Queen's University Belfast Research Portal is retained by the author(s) and / or other copyright owners and it is a condition of accessing these publications that users recognise and abide by the legal requirements associated with these rights.

Take down policy

The Research Portal is Queen's institutional repository that provides access to Queen's research output. Every effort has been made to ensure that content in the Research Portal does not infringe any person's rights, or applicable UK laws. If you discover content in the Research Portal that you believe breaches copyright or violates any law, please contact openaccess@qub.ac.uk. 


\title{
Pilot Power Control for Cell-free Massive MIMO
}

\author{
Trang C. Mai, Hien Quoc Ngo, Malcolm Egan, and Trung Q. Duong, Senior Member, IEEE,
}

\begin{abstract}
In this paper, we consider a cell-free massive MIMO system where access points (APs) serve a much smaller number of users under the time-division duplex operation. The APs first estimate the channels via the uplink training phase. Then these channel estimates are used to detect desired symbols in the uplink and precode the transmit symbols in the downlink. Nonorthogonality of pilot sequences and AP selection (e.g. receivedpower-based selection or largest-large-scale-fading based selection schemes) are taken into account. To reduce the effect of pilot contamination, we propose a pilot power control design which chooses the pilot power control coefficients to minimize the meansquared error of the channel estimation. This is achieved via the sequential convex approximation method. By using pilot power control in training phase, the system performance is considerably improved. In addition, we derive closed-form expressions for the uplink and downlink achievable rates with arbitrary power data/pilot control coefficients and any AP selection schemes.
\end{abstract}

Index terms - Cell-free massive MIMO, channel estimation, pilot power control.

\section{INTRODUCTION}

Cell-free massive MIMO systems, where a large number of access points (APs) serve a much smaller number of users in the network simultaneously, in the same frequency resource, have attracted significant attention as they provides uniformly good service to all users as well as handover-free [1], [2]. Moreover, by using multiple antennas at APs, cell-free massive MIMO inherits the important benefit, i.e. channel hardening, from massive MIMO [3].

In cell-free systems, all channels estimated in the training phase are used for precoding in the downlink data transmission and decoding in the uplink data transmission. In the training phase, each user should ideally be assigned a pilot sequence which is mutually orthogonal to other users' pilot sequences. However, when the coherence interval is short or/and the number of users in the system is large [4], non-orthogonal pilot sequences have to be used by the users. As a consequence, at each AP, the pilot signal transmitted from a given user will be interfered by the pilot signal transmitted from other users. This effect, known as pilot contamination, reduces the system performance significantly [5], [6]. To mitigate pilot contamination, pilot assignment and data power controls can be used [1], [7].

In previous works on cell-free massive MIMO, all pilot signals are transmitted with full power during the training phase [1], [3], [7]-[9]. It may happen that a user with poor channels can be strongly contaminated by users with strong channels during the training phase. This significantly degrades

T. C. Mai, H. Q. Ngo, and T. Q. Duong are with the Institute of Electronics, Communications and Information Technology in Queen's University Belfast, Belfast, U.K. (email: \{tmai01, hien.ngo, trung.q.duong\}@qub.ac.uk)

M. Egan is with Univ Lyon, INSA Lyon, INRIA, CITI, France (email: malcolm.egan@gmail.com ) the performance of the user, and hence, reduces the total performance of the systems. In addition, [7] shows that, it is not optimal to use all APs to serve all users in both uplink and downlink data transmission phases, especially when taking into account the capacity limitation of backhaul load [9] and the hardware impairments [8]. Therefore, in this work we propose to allocate pilot power for each user to improve the channel estimation quality. We consider a cell-free massive MIMO system as in [1], but the APs are equipped with multiple antennas. In particular, the power control and AP selection are exploited during the training phase. In general, we should assign the pilot sequences, allocate the pilot powers, and select the number of serving APs per user to maximize the system performance. However, this is a multi-objective optimization problem and finding the corresponding optimal solutions is a challenging task. Therefore, in this work we focus on allocating the pilot powers, assuming that pilot assignments are designed in advance. Cell-free massive MIMO utilizes channel state information that is estimated rather than assumed and hence the accuracy of channel estimation is very important.

The objective in this paper is to minimize the channel estimation error. More precisely, we propose a simple pilot power control problem which can improve the net throughput via minimizing the variance of the MMSE channel estimation error. The main contributions of this paper are as follows

- We derive closed-form expressions for both uplink and downlink achievable rates of a cell-free massive MIMO system taking into account the channel estimate errors, AP selection schemes, multiple antennas at the APs, and pilot/data power controls. Our result is a generalization of the result in [1] in which pilot power control, multiple antennas at the APs, and AP selection were not considered.

- We propose a pilot power control problem which aims at minimizing the mean-squared channel estimation errors, subject to a pilot power constraint. The proposed optimization problem is not convex, but it can be changed to a convex problem by using the second-order Taylor approximation. As a result, its solution can be approximately determined by solving a sequence of convex programs.

\section{System Model and SPECTRAL EFFiciency}

We consider a cell-free massive MIMO system where $M$ geographically distributed APs jointly serve all $K$ users in the same time-frequency resource of the network. Each user is served by a subset of APs. Let $\mathcal{A}_{k}$ be the set of APs serving the $k$-th user and $\mathcal{U}_{m}$ be the set of users served by the $m$-th 
AP. ${ }^{1}$ The system operates in TDD mode and exploits channel reciprocity. The channels estimated at the APs can be used to decode the signals transmitted from the users in the uplink, and precode the downlink symbols. Each AP has $L$ antennas, while each user is equipped with a single-antenna. The channel vector, $\mathbf{g}_{m k} \in \mathbb{C}^{L \times 1}$, between the $k$-th user and the $m$-th AP is modeled as the combination of the large-scale fading $\beta_{m k}$ and the vector of small-scale fading $\mathbf{h}_{m k} \in \mathbb{C}^{L \times 1}$ :

$$
\mathbf{g}_{m k}=\beta_{m k}^{1 / 2} \mathbf{h}_{m k} .
$$

We assume that $\left\{\mathbf{h}_{m k}\right\}, k=1, \ldots, K, m=1, \ldots, M$, are independent and identically distributed (i.i.d.) $\mathcal{C N}\left(0, \mathbf{I}_{L}\right)$ random variable (RV) vectors, and $\beta_{m k}$ are known a priori.

For each coherence interval, the transmission occurs in three phases: uplink training, uplink data transmission, and downlink data transmission. The system model is similar to the one in [1]. The main difference appears in the training phase. More precisely, in [1] the users transmit with full powers during the training phase, while here in our work, the pilot signals will be weighted by the power control coefficients. The power control is done at the CPU. As in previous works [1], we assume that the power control coefficients are sent to the APs perfectly via dedicated channels. Note that this is done for each large-scale fading realization which changes very slowly.

\section{A. Uplink Training Phase}

For each coherence interval of length $\tau_{\mathrm{c}}$ (symbols), a duration of length $\tau$ (symbols) is used for the uplink training. Let $\sqrt{\tau \eta_{k}} \boldsymbol{\varphi}_{k} \in \mathbb{C}^{\tau \times 1}$, where $\left\|\varphi_{k}\right\|^{2}=1$, be the pilot sequence of user $k$. Here, $\eta_{k}$, where $0<\eta_{k} \leq 1$, be the corresponding power control coefficient. Then, the $m$-th AP receives

$$
\mathbf{Y}_{\mathrm{p}, m}=\sqrt{\tau \rho_{\mathrm{p}}} \sum_{k=1}^{K} \mathbf{g}_{m k} \eta_{k}^{1 / 2} \boldsymbol{\varphi}_{k}^{H}+\mathbf{W}_{\mathrm{p}, m}
$$

where $\rho_{\mathrm{p}}$ is the normalized (with respect to noise power) signal-to-noise ratio ( $\mathrm{SNR}$ ) of each pilot symbol, $\mathbf{W}_{\mathrm{p}, m}$ is the $L \times \tau_{c}$ additive noise matrix that their elements are i.i.d. $\mathcal{C N}(0,1)$ RVs. After projecting $\mathbf{Y}_{\mathrm{p}, m}$ onto $\varphi_{k}^{H}$, the MMSE estimate of $\mathbf{g}_{m k}$ is

$$
\begin{aligned}
\hat{\mathbf{g}}_{m k} & =\mathbb{E}\left\{\mathbf{g}_{m k} \check{\mathbf{y}}_{\mathrm{p}, m k}^{H}\right\}\left(\mathbb{E}\left\{\check{\mathbf{y}}_{\mathrm{p}, m k} \check{\mathbf{y}}_{\mathrm{p}, m k}^{H}\right\}\right)^{-1} \check{\mathbf{y}}_{\mathrm{p}, m k} \\
& =c_{m k} \check{\mathbf{y}}_{\mathrm{p}, m k},
\end{aligned}
$$

where $\check{\mathbf{y}}_{\mathrm{p}, m k} \triangleq \mathbf{Y}_{\mathrm{p}, m} \boldsymbol{\varphi}_{k}$, and

$$
c_{m k} \triangleq \frac{\sqrt{\tau \rho_{\mathrm{p}}} \beta_{m k} \eta_{k}^{1 / 2}}{\tau \rho_{\mathrm{p}} \sum_{k^{\prime}=1}^{K} \beta_{m k^{\prime}} \eta_{k^{\prime}}\left|\boldsymbol{\varphi}_{k}^{H} \boldsymbol{\varphi}_{k^{\prime}}\right|^{2}+1} .
$$

$\gamma_{m k}$ denotes the $l$-th component's mean-square of the estimated channel vector $\hat{\mathbf{g}}_{m k}$, can be calculated as

$$
\gamma_{m k} \triangleq \mathbb{E}\left\{\left|\left[\hat{\mathbf{g}}_{m k}\right]_{l}\right|^{2}\right\}=\sqrt{\tau \rho_{\mathrm{p}}} \beta_{m k} \eta_{k}^{1 / 2} c_{m k},
$$

where $[.]_{l}$ denotes $l$-th component of the vector [.].

\footnotetext{
${ }^{1} \mathrm{AP}$ selection is applied to reduce the requirement for backhaul connection In a special case where $\mathcal{A}_{k}=\{1,2, \ldots, M\}$, for all $k$, we have the same system model as the one in [1]. The AP selection can be done by some criterion such as received-power-based selection or largest-large-scale-fadingbased selection schemes in [7].
}

\section{B. Uplink Data Transmission}

All $K$ users share the same time-frequency resource. The received signal at the $m$-th AP is modeled as

$$
\mathbf{y}_{\mathrm{u}, m}=\sqrt{\rho_{\mathrm{u}}} \sum_{k=1}^{K} \mathbf{g}_{m k} \sqrt{\eta_{k}^{\mathrm{u}}} q_{k}+\mathbf{w}_{\mathrm{u}, m},
$$

where $q_{k}, \mathbb{E}\left\{\left|q_{k}\right|^{2}\right\}=1$, is the symbol of the $k$-th user, $\eta_{k}^{\mathrm{u}}$, $0 \leq \eta_{k}^{\mathrm{u}} \leq 1$, is the data power control coefficient, $\rho_{\mathrm{u}}$ is the normalized uplink $\mathrm{SNR}$, and $w_{\mathrm{u}, m} \sim \mathcal{C N}(0,1)$ is the additive noise.

To detect the symbol transmitted from the $k$-th user, the $m$-th AP which belongs to the subset $\mathcal{A}_{k}$ sends $\hat{g}_{m k}^{*} y_{\mathrm{u}, m}$ to the CPU through the backhaul network. The received signal at the CPU is

$$
r_{\mathrm{u}, k}=\sum_{m \in \mathcal{A}_{k}} \sum_{l=1}^{L}\left[\hat{\mathbf{g}}_{m k}\right]_{l}^{*}\left[\mathbf{y}_{\mathrm{u}, m}\right]_{l} .
$$

Following the same technique in [1], we first decompose the received signal $r_{\mathrm{u}, k}$ as

$$
r_{\mathrm{u}, k}=\mathrm{DS}_{k} \cdot q_{k}+\mathrm{BU}_{k} \cdot q_{k}+\sum_{k^{\prime} \neq k}^{K} \mathrm{UI}_{k k^{\prime}} \cdot q_{k^{\prime}}+\mathrm{N},
$$

where

$$
\begin{aligned}
& \mathrm{DS}_{k} \triangleq \sqrt{\rho_{\mathrm{u}} \eta_{k}^{u}} \mathbb{E}\left\{\sum_{m \in \mathcal{A}_{k}} \sum_{l=1}^{L}\left[\hat{\mathbf{g}}_{m k}\right]_{l}^{*}\left[\mathbf{g}_{m k}\right]_{l}\right\}, \\
& \mathrm{BU}_{k} \triangleq \sqrt{\rho_{\mathrm{u}} \eta_{k}^{u}} \sum_{m \in \mathcal{A}_{k}} \sum_{l=1}^{L}\left[\hat{\mathbf{g}}_{m k}\right]_{l}^{*}\left[\mathbf{g}_{m k}\right]_{l}-\mathrm{DS}_{k}, \\
& \mathrm{UI}_{k k^{\prime}} \triangleq \sqrt{\rho_{\mathrm{u}} \eta_{k^{\prime}}^{u}} \sum_{m \in \mathcal{A}_{k}} \sum_{l=1}^{L}\left[\hat{\mathbf{g}}_{m k}\right]_{l}^{*}\left[\mathbf{g}_{m k^{\prime}}\right]_{l}, \\
& \text { and } \mathrm{N} \triangleq \sum_{m \in \mathcal{A}_{k}} \sum_{l=1}^{L}\left[\hat{\mathbf{g}}_{m k}\right]_{l}^{*}\left[\mathbf{w}_{\mathrm{u}, m}\right]_{l} .
\end{aligned}
$$

Then by treating the sum of the second, third, and fourth terms in (8) as effective noise and using the worst-case Gaussian noise argument [10], we obtain the spectral efficiency of the $k$-th user as follows:

$$
\begin{aligned}
& S_{\mathrm{u}, k}=\frac{1-\tau / \tau_{\mathrm{c}}}{2} \times \\
& \times \log _{2}\left(1+\frac{\left|\mathrm{DS}_{k}\right|^{2}}{\mathbb{E}\left\{|\mathrm{BU}|^{2}\right\}+\sum_{k^{\prime} \neq k}^{K} \mathbb{E}\left\{\left|\mathrm{UI}_{k k^{\prime}}\right|^{2}\right\}+\mathbb{E}\left\{|\mathrm{N}|^{2}\right\}}\right) .
\end{aligned}
$$

Following the derivation in Appendix A, we can obtain the closed-form expression for the uplink spectral efficiency (9) as given in (13), shown at the top of the next 2 pages.

\section{Downlink Data Transmission}

In the downlink, the APs belonging to the set $\mathcal{A}_{k}$ aim to send symbol $q_{k}$, where $\mathbb{E}\left\{\left|q_{k}\right|^{2}\right\}=1$, to user $k, k=$ $1, \ldots, K$. As a result, $m$-th AP will aim to send symbols to the users in the set $\mathcal{U}_{m}$. The vector of transmitted signals from the $m$-th AP, which uses maximum ratio precoding scheme 
based on its local channel estimates to precode the transmitted symbols, is

$$
\mathbf{x}_{m}=\sqrt{\rho_{\mathrm{d}}} \sum_{k \in \mathcal{U}_{m}} \sqrt{\eta_{m k}^{d}} \hat{\mathbf{g}}_{m k}^{*} q_{k}
$$

where $\rho_{\mathrm{d}}$ is the normalized downlink SNR, $\eta_{m k}^{d}$ are the downlink power control coefficients chosen to satisfy the power constraint at each AP $\mathbb{E}\left\{\left\|x_{m}\right\|^{2}\right\} \leq \rho_{\mathrm{d}}$, and hence,

$$
\sum_{k \in \mathcal{U}_{m}} \eta_{m k}^{d} \gamma_{m k} \leq \frac{1}{L}, \quad \forall m
$$

The received signal at the $k$-th user is

$$
r_{\mathrm{d}, k}=\sqrt{\rho_{\mathrm{d}}} \sum_{m=1}^{M} \sum_{k^{\prime} \in \mathcal{U}_{m}} \sqrt{\eta_{m k^{\prime}}^{d}} \mathbf{g}_{m k}^{T} \hat{\mathbf{g}}_{m k^{\prime}}^{*} q_{k^{\prime}}+w_{\mathrm{d}, k}
$$

where $w_{\mathrm{d}, m} \sim \mathcal{C N}(0,1)$ is additive noise at the $k$-th user.

Following a similar methodology as the derivation of the uplink spectral efficiency in Section II-B, we obtain closedform expression for the downlink spectral efficiency as given by (14), shown at the top of the next page.

\section{Pilot Power Control}

The cell-free massive MIMO system operates in TDD mode and the channels are estimated at each AP based on the pilot signals sent from the users during the training phase. Due to the non-orthogonality among pilot sequences, the estimate of the channel corresponding to a given user will be impaired by the pilots transmitted from other users. This effect is known as pilot contamination effect. This effect is very serious in cellfree massive MIMO systems, where we aim to serve many users simultaneously, in the same time-frequency resource. Therefore, pilot contamination mitigation is crucial task in cell-free massive MIMO. To reduce the pilot contamination effect, we propose to design the pilot power coefficients to improve the channel estimation accuracy during the training phase. Since we aim at providing uniformly good service for all users in the network, we propose a min-max optimization problem which minimizes the largest of all user normalized mean-squared errors (normalized by its large-scale fading coefficient) as follows

$$
\begin{aligned}
& \min _{\left\{\eta_{k}\right\}} \max _{k=1, \cdots, K} \sum_{m \in \mathcal{A}_{k}} \frac{\operatorname{var}_{m k}}{\beta_{m k}} \\
& \text { subject to } \epsilon \leq \eta_{k} \leq 1, \quad \forall k=1, \ldots, K,
\end{aligned}
$$

where $\mathcal{A}_{k}$ is again the set of APs chosen to serve the $k$-th user, $\epsilon$ is very small and $>0$ (this guarantees that all required channels are estimated in the training phase), and $\operatorname{var}_{m k}$ is the mean-squared error of MMSE estimation for $\left[\mathbf{g}_{m k}\right]_{l}$ given by [11]

$$
\operatorname{var}_{m k}=\beta_{m k}-\gamma_{m k}=\beta_{m k}\left(1-\frac{\gamma_{m k}}{\beta_{m k}}\right)
$$

An equivalent form of (15) is

$\min _{\left\{\eta_{k}\right\}, t} t$

subject to

$$
\begin{aligned}
& t \geq \sum_{m \in \mathcal{A}_{k}}\left(1-\frac{\tau \rho_{\mathrm{p}} \beta_{m k} \eta_{k}}{\tau \rho_{\mathrm{p}} \sum_{k^{\prime}=1}^{K} \beta_{m k^{\prime}} \eta_{k^{\prime}}\left|\boldsymbol{\varphi}_{k}^{H} \boldsymbol{\varphi}_{k^{\prime}}\right|^{2}+1}\right), \forall k \\
& \epsilon \leq \eta_{k} \leq 1, \quad \forall k .
\end{aligned}
$$

This is a non-convex problem. To solve this, we use the successive approximation technique with first order Taylor approximation for the convex function $\frac{1}{x y}$ with $x, y>0$ as follows

$$
\frac{1}{x y} \geq \frac{3}{x_{0} y_{0}}-\frac{x}{x_{0}^{2} y_{0}}-\frac{y}{x_{0} y_{0}^{2}}
$$

We apply the approximation in (18) for each fraction in the right-hand side of (17b), where $x_{m k}=\frac{1}{\tau \rho_{\mathrm{p}} \beta_{m k} \eta_{k}}$ and $y_{m k}=$ $\tau \rho_{\mathrm{p}} \sum_{k^{\prime}=1}^{K} \beta_{m k^{\prime}} \eta_{k^{\prime}}\left|\boldsymbol{\varphi}_{k}^{H} \boldsymbol{\varphi}_{k^{\prime}}\right|^{2}+1$ with $\forall k=1, \ldots, K$ and $m \in \mathcal{A}_{k}$. The solution of problem (17) can be upper bounded by solution of a problem

$\min _{\left\{\eta_{k}\right\}, t} t$

subject to

$$
\begin{aligned}
& t \geq \sum_{m \in \mathcal{A}_{k}}\left(1-\frac{3}{x_{m k 0} y_{m k 0}}+\frac{x_{m k}}{x_{m k 0}^{2} y_{m k 0}}+\frac{y_{m k}}{x_{m k 0} y_{m k 0}^{2}}\right), \forall k, \\
& \epsilon \leq \eta_{k} \leq 1, \quad \forall k .
\end{aligned}
$$

Finally, problem (15) can be determined by solving a sequence of the convex problem (19). The details are shown in Algorithm 1 .

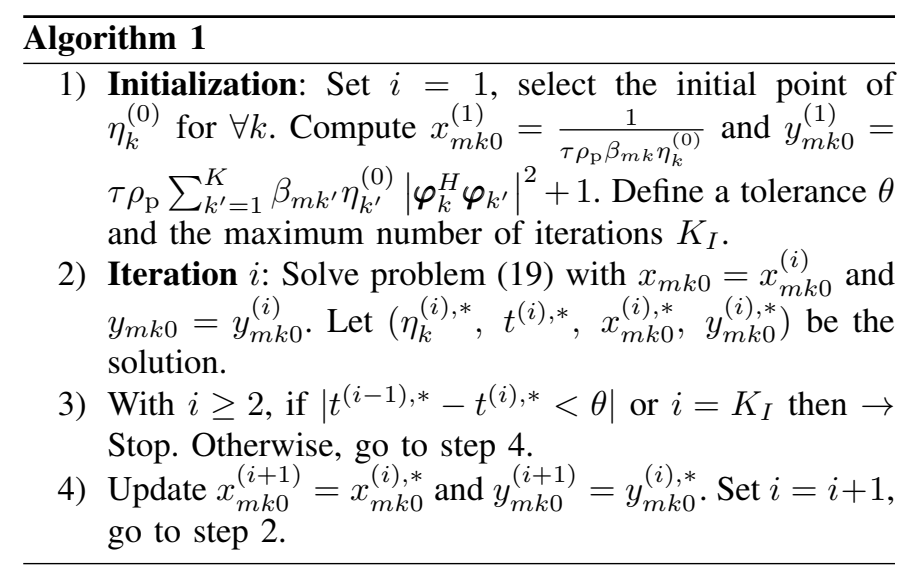

Convergence Analysis: The convergence analysis follows the general framework in [12]. An optimal solution of (19) is also feasible to (15) as the result of convex bounds in above successive approximation technique. Therefore, Algorithm 1 yields a non-increasing sequence of objectives. Moreover, due to the power constraints, the objective of (19) is bounded from below. Thus the objective of Algorithm 1 is guaranteed to converge. 
$S_{\mathrm{u}, k}=\frac{1-\tau / \tau_{\mathrm{c}}}{2} \log _{2}\left(1+\frac{L^{2} \rho_{\mathrm{u}} \eta_{k}^{u}\left(\sum_{m \in \mathcal{A}_{k}} \gamma_{m k}\right)^{2}}{L^{2} \rho_{\mathrm{u}} \sum_{k^{\prime} \neq k}^{K} \eta_{k^{\prime}}^{u}\left|\boldsymbol{\varphi}_{k}^{H} \boldsymbol{\varphi}_{k^{\prime}}\right|^{2}\left(\sum_{m \in \mathcal{A}_{k}} \gamma_{m k} \sqrt{\frac{\eta_{k}^{\prime}}{\eta_{k}} \frac{\beta_{m k^{\prime}}}{\beta_{m k}}}\right)^{2}+L \rho_{\mathrm{u}} \sum_{k^{\prime}=1}^{K} \eta_{k^{\prime}}^{u} \sum_{m \in \mathcal{A}_{k}} \gamma_{m k} \beta_{m k^{\prime}}+L \sum_{m \in \mathcal{A}_{k}} \gamma_{m k}}\right)$

$S_{\mathrm{d}, k}=\frac{1-\tau / \tau_{\mathrm{c}}}{2} \log _{2}\left(1+\frac{L^{2} \rho_{\mathrm{d}}\left(\sum_{m \in \mathcal{A}_{k}} \sqrt{\eta_{m k}^{d}} \gamma_{m k}\right)^{2}}{L^{2} \rho_{\mathrm{d}} \sum_{k^{\prime} \neq k}^{K}\left|\varphi_{k^{\prime}}^{H} \boldsymbol{\varphi}_{k}\right|^{2}\left(\sum_{m \in \mathcal{A}_{k^{\prime}}} \sqrt{\eta_{m k^{\prime}}^{d}} \gamma_{m k^{\prime}} \sqrt{\frac{\eta_{k}}{\eta_{k^{\prime}}}} \frac{\beta_{m k}}{\beta_{m k^{\prime}}}\right)^{2}+L \rho_{\mathrm{d}} \sum_{k^{\prime}=1}^{K} \sum_{m \in \mathcal{A}_{k^{\prime}}} \eta_{m k^{\prime}}^{d} \gamma_{m k^{\prime}} \beta_{m k}+1}\right)$,

\section{NumERiCAL Results AND Discussions}

In this section, we compare per-user net throughputs of our proposed pilot power control method with the ones with full pilot power. In all examples, max-min power control in the data transmission phase proposed in [1] is applied. We choose $M=100$ and $K=40$. For simplicity, we use the largestlarge-scale-fading-based selection scheme in [7] to choose the set of APs $\mathcal{A}_{k}$, and denote by $N=\left|\mathcal{A}_{k}\right|$. Furthermore, random pilot assignment is used, i.e., each user randomly selects a pilot sequence from a predefined set of orthogonal pilot sequences.

\section{A. Parameters and Setup}

The paper metrics and setup resemble those of [1]. More precisely, APs and users randomly locate on a square, and wrapped around technique is applied to imitate a network with an infinite area. The large-scale fading coefficient $\beta_{m k}$ is modeled by the path loss and uncorrelated log-normal shadowing. We use three-slope model to generate the path loss. Then, the per-user net throughput, which is considered as the quality metric of the system, is given by

$$
T_{\mathrm{Ch}, k}=B S_{\mathrm{Ch}, k},
$$

where $\mathrm{Ch} \in\{\mathrm{u}, \mathrm{d}\}$ corresponds to uplink or downlink, respectively, and $B$ is the spectral bandwidth. Numerical results are obtained by 500 random realizations of AP and user locations.

\section{B. Results and Discussions}

We first examine the convergence behavior of Algorithm 1. Figure 1 shows the normalized mean-squared error (the objective function of (15)) versus the number of iterations with different $\tau$ and $N$, for an arbitrary snapshot of large-scale fading realization. We can see that our algorithm converges very fast, just after 5 iterations.

Next, we evaluate the effectiveness of proposed pilot power control. Note that our proposed method is based on the successive approximation technique in Algorithm 1, and hence, it yields sub-optimal results. Figures 2 and 3 show the cumulative distribution (CD) of the per-user uplink net throughputs for $\tau=5$ and $\tau=10$. While Figures 4 and 5 show the corresponding $\mathrm{CD}$ of the per-user downlink net throughputs. In our pilot power control algorithm, we choose $\epsilon=0.01$, $\theta=0.005$, and $K_{I}=15$. Note that the "no power control" curves correspond to the cases where the users transmit with full power during the training phase [1].

Numerical results show that our pilot power control algorithm outperforms the previous method of [1] in both uplink and downlink transmissions, especially for 95\%-likely throughputs. Compared to [1], our proposed method offers more performance improvement in the cases that $\tau$ and $N$ are small, i.e. $\tau=5$ and $N=5$. As shown in Figure 2 and Figure 4, our proposed method offers over $20 \%$ improvement in the uplink net throughput and over $25 \%$ improvement in the downlink net throughput with $N=5$ serving APs per user and $\tau=5$. The reason comes from the fact that at low $\tau$ and $N$, the system suffers more pilot contamination. Our proposed scheme can reduce this pilot contamination effect.

With a fixed number of serving APs per user, if $\tau$ is small, longer time per coherence interval is spent for the data transmission, but the performance of channel estimation is poor as it suffers more pilot contamination. By contrast, if $\tau$ is large, the performance of channel estimation is better but smaller time per coherence interval is spent for data transmission. However, regardless of $\tau$, the system performance with pilot power control always outperforms the case without pilot power control.

Numerical results also show that the system throughput improves when we increase the number of serving APs per user as well as the number of antennas per AP. However, the system performance need to be traded off among some parameter constraints such as the capacity of backhaul load, energy efficiency, and throughput of the system. We leave this problem for the future work.

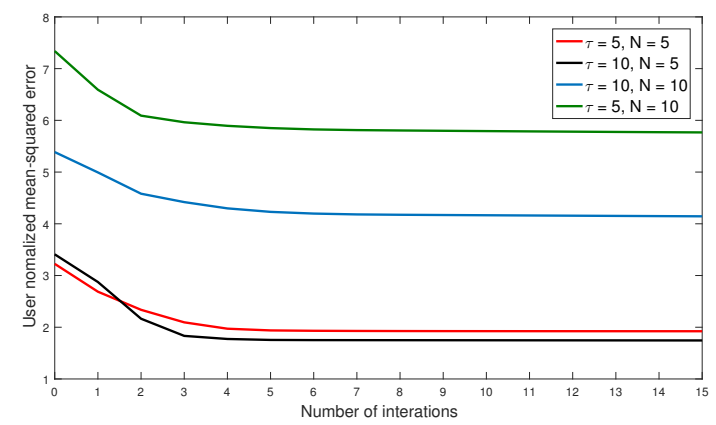

Fig. 1. User nomalized mean-squared error vs number of iterations. 


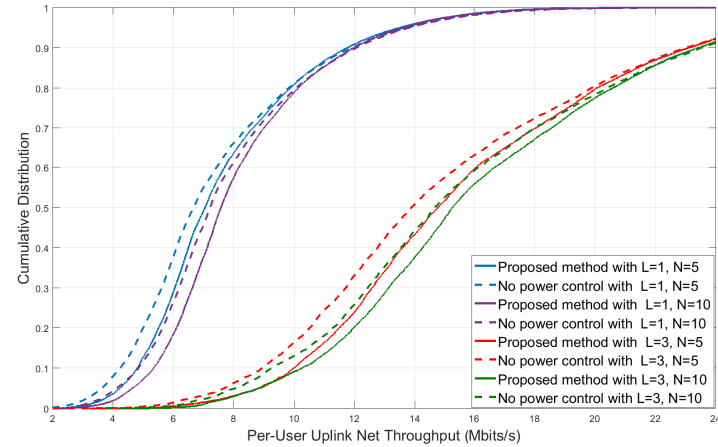

Fig. 2. $\mathrm{CD}$ of the per-user uplink net throughput with $\tau=5$.

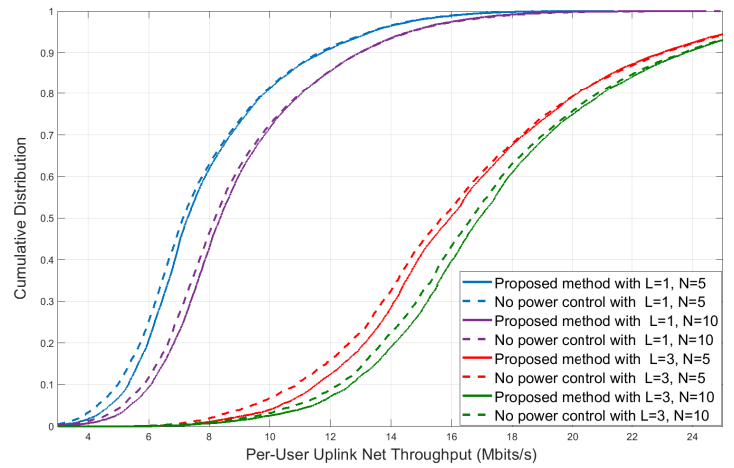

Fig. 3. CD of the per-user uplink net throughput with $\tau=10$.

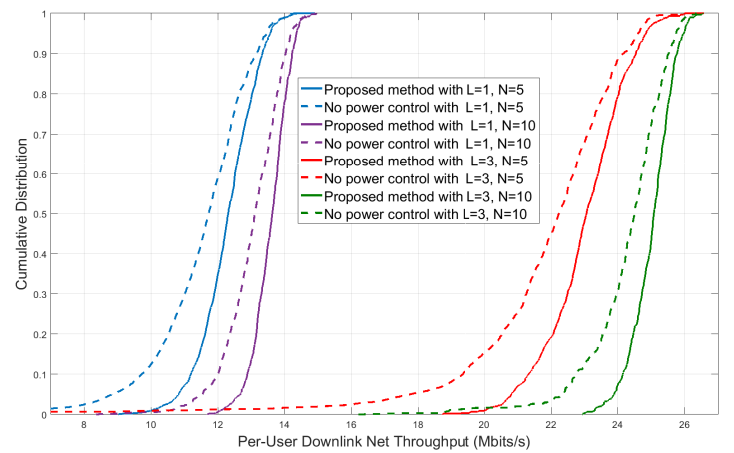

Fig. 4. CD of the per-user downlink net throughput with $\tau=5$.

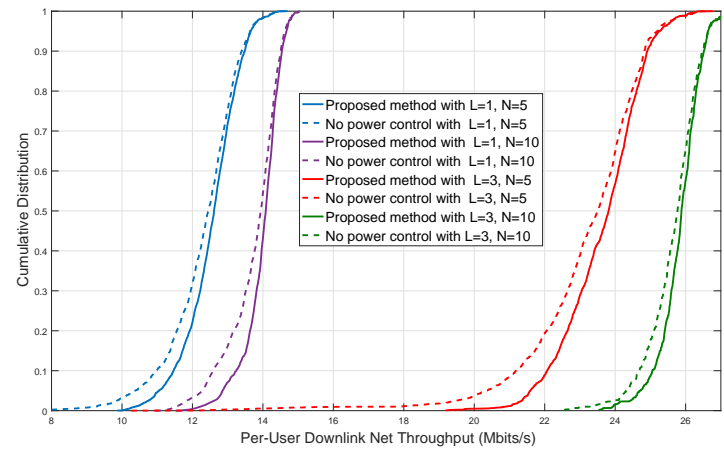

Fig. 5. CD of the per-user downlink net throughput with $\tau=10$.

\section{CONCLUSION}

Typically, in cell-free massive MIMO, the users transmit with full power during the training phase. In this paper we exploited how much would the performance improve if the pilot powers is optimally chosen. We proposed to design the pilot power control coefficients to improve the channel estimation quality, and hence, reduce the effect of pilot contamination. Compared to the case without pilot power control, our proposed method offered a noticeable throughput improvement in both uplink and downlink. We also found that the proposed pilot power control scheme is preferable for highmobility environments (i.e. $\tau_{c}$ is small).

\section{APPENDIX}

\section{A. Derivation of (13)}

Following the same technique in [1], we have

$$
\begin{aligned}
\mathrm{DS}_{k} & =L \sqrt{\rho_{\mathrm{u}} \eta_{k}^{u}} \sum_{m \in \mathcal{A}_{k}} \gamma_{m k}, \\
\mathbb{E}\left\{\left|\mathrm{BU}_{k}\right|^{2}\right\} & =L \rho_{\mathrm{u}} \eta_{k}^{u} \sum_{m \in \mathcal{A}_{k}} \gamma_{m k} \beta_{m k},
\end{aligned}
$$

and

$$
\begin{aligned}
\mathbb{E}\left\{\left|\mathrm{UI}_{k k^{\prime}}\right|^{2}\right\}= & L^{2} \rho_{\mathrm{u}} \eta_{k^{\prime}}^{u}\left|\boldsymbol{\varphi}_{k}^{H} \boldsymbol{\varphi}_{k^{\prime}}\right|^{2}\left(\sum_{m \in \mathcal{A}_{k}} \gamma_{m k} \sqrt{\frac{\eta_{k}^{\prime}}{\eta_{k}}} \frac{\beta_{m k^{\prime}}}{\beta_{m k}}\right)^{2} \\
& +L \rho_{\mathrm{u}} \eta_{k^{\prime}}^{u} \sum_{m \in \mathcal{A}_{k}} \gamma_{m k} \beta_{m k^{\prime}}
\end{aligned}
$$

Finally, (13) is obtained by substitution of (21), (22) and (23) into (9).

\section{REFERENCES}

[1] H. Q. Ngo, A. Ashikhmin, H. Yang, E. G. Larsson, and T. L. Marzetta, "Cell-free massive MIMO versus small cells," IEEE Trans. Wireless Commun., vol. 16, no. 3, pp. 1834-1850, Mar. 2017.

[2] S. Buzzi and C. D'Andrea, "Cell-free massive MIMO: User-centric approach," IEEE Wireless Commun. Lett., vol. 6, no. 6, pp. 706-709, Aug. 2017.

[3] Z. Chen and E. Björnson, "Can we rely on channel hardening in cell-free massive MIMO?" in Proc. IEEE GLOBECOM Workshops, Singapore, Dec. 2017, pp. 1-6.

[4] E. Björnson, E. G. Larsson, and M. Debbah, "Massive MIMO for maximal spectral efficiency: How many users and pilots should be allocated?" IEEE Trans. Wireless Commun., vol. 15, no. 2, pp. 12931308, Feb. 2016.

[5] T. L. Marzetta, "Noncooperative cellular wireless with unlimited numbers of base station antennas," IEEE Trans. Wireless Commun., vol. 9, no. 11, pp. 3590-3600, Nov. 2010.

[6] N. Akbar, S. Yan, N. Yang, and J. Yuan, "Location-aware pilot allocation in multicell multiuser massive MIMO networks," IEEE Trans. Veh. Technol., vol. 67, no. 8, pp. 7774-7778, Aug. 2018.

[7] H. Q. Ngo, L. Tran, T. Q. Duong, M. Matthaiou, and E. G. Larsson, "On the total energy efficiency of cell-free massive MIMO," IEEE Trans. Green Commun. Netw., vol. 2, no. 1, pp. 25-39, Mar. 2018.

[8] J. Zhang, Y. Wei, E. Björnson, Y. Han, and X. Li, "Spectral and energy efficiency of cell-free massive MIMO systems with hardware impairments," in Proc. IEEE WCSP, Nanjing, China, Oct. 2017, pp. $1-6$.

[9] M. Bashar, K. Cumanan, A. G. Burr, H. Q. Ngo, and M. Debbah, "Cellfree massive MIMO with limited backhaul," in Proc. IEEE ICC, MO, USA, May 2018, pp. 1-7.

[10] B. Hassibi and B. M. Hochwald, "How much training is needed in multiple-antenna wireless links?" IEEE Trans. Inf. Theory, vol. 49, no. 4, pp. 951-963, April 2003.

[11] S. M. Kay, Fundamentals of statistical signal processing: Estimation theory. Upper Saddle River, NJ, USA: Prentice Hall, 1993.

[12] A. Beck, A. Ben-Tal, and L. Tetruashvili, "A sequential parametric convex approximation method with applications to nonconvex truss topology design problems," J. Glob. Optim., vol. 47, no. 1, pp. 29-51, May 2010. 